\title{
LA IGUALDAD ENTRE GÉNEROS Y LA NECESIDAD DE UN NUEVO PRINCIPIO DE ACCIÓN. APRENDIZAJE Y PROFESIONALIZACIÓN EN LA EDUCACIÓN SUPERIOR
}

\author{
$\mathrm{M}^{\mathrm{a}}$ Isabel Lafuente Guantes \\ Universidad de León \\ milafg@unileon.es
}

Recibido: 24-11-2010

Aceptado: 12-04-2011

\section{Resumen}

Se muestra la marginación en que se halla la mujer en el campo profesional de la educación superior a partir de las principales acciones realizadas sobre esta situación y su análisis cuantitativo en la actualidad. Saber de qué depende la marginación y cómo puede contrarrestarse requiere: 1. Entender la noción de lucha contra el tiempo. 2. Conocer el alcance que tiene el principio ilustrado de igualdad educativa.3. Saber de qué principios depende la marginación de la mujer. 4. Establecer la forma de variar estos principios en conformidad con los valores propios de la mujer.

Palabras Clave: Mujer, educación superior, lucha contra el tiempo, igualdad, fuerza mayor, diálogo.

\begin{abstract}
This paper shows the marginalization of women in the professional field of university education, on the basis of the principal actions undertaken on this situation and the quantitative analysis thereof at present. In order to know on what marginalization depends and how it can be counteracted, it is required: 1.To understand the notion of fight against time. 2. To know the scope of the illustrated principle of equal education. 3. To know from what principles the marginalization of women depends. 4. To establish the way of changing these principles in conformity with women's own values.
\end{abstract}

Keywords: Woman, university education, fights against time, equality, force majeure, dialog. 


\section{La herencia recibida en la lucha por los derechos de la mujer en la educación superior}

Desde finales del siglo pasado ha ido aumentando, y de forma muy considerable, la preocupación por visualizar, hacer conocer y remediar la situación de injusticia de la mujer en la educación superior, tanto en los estudios como en la carrera docente y en la investigación.

Educativamente la marginación de la mujer en España ha sido innegable. A principios del siglo XX todas las mujeres prácticamente eran analfabetas, y las poquísimas que se atrevían a asistir a las universidades lo hacían disfrazando su condición femenina, pues hasta 1910 (Real Orden de 8 de marzo) no se autoriza el libre acceso de la mujer a la enseñanza superior.

Actualmente la situación ha cambiado de forma radical en lo que afecta al acceso a la educación universitaria y al proceso de aprendizaje, pero no en lo relativo a la profesionalización: investigación, acceso a plazas docentes superiores y a órganos de gobierno. Bien es cierto que existe una Ley de Igualdad general, que en el caso de la Ley de Universidades garantiza la presencia equilibrada entre hombres y mujeres en los órganos de gobierno. Pero, en la Orden PRE/525/2005, publicada en el B.O.E., se reconoce la necesidad de adoptar medidas que favorezcan la igualdad entre hombres y mujeres dado que:

“[...] la discriminación histórica de las mujeres no se ha erradicado en una sociedad que sigue sin ofrecer iguales oportunidades a todos los ciudadanos y ciudadana".

La atención al problema de la mujer en la educación superior es muy reciente. En Europa es entre 1988 y 1999 cuando tienen lugar diversas acciones que inciden en el problema de la situación de la mujer en la ciencia. A partir de 1996 los informes empiezan a poner de relieve los puntos débiles existentes en la igualdad de género y, en ese contexto, se señala la mayor incidencia de la mujer en los estudios de las áreas de letras, humanidades y ciencias sociales, y su poca, o prácticamente nula, presencia en las de ciencia y tecnología.

De forma paralela a las acciones en favor de la igualdad de los géneros en otros países, en España se aprobó por la Junta de Gobierno y el Consejo Social de la Universidad Complutense de Madrid la creación de un Instituto de Investigaciones Feministas, que sirvió de apoyo a la creación de Seminarios, Cátedras y Centros de Investigación Feminista en otras universidades. Estas acciones culminan en 2005 con la 
creación de la Unidad de Mujer y Ciencia (UMICYT), a la que se marcan los objetivos de elaborar datos segregados por sexo y realizar acciones positivas para evitar la discriminación y, en 2008, durante el gobierno de Rodríguez Zapatero, con la creación del Ministerio de Igualdad, del que depende como organismo autónomo, a través del Secretariado de Políticas de Igualdad, el Instituto de la Mujer ${ }^{1}$. En octubre del año 2010, este Ministerio ha sido suprimido; sólo queda la esperanza de que perduren las políticas de igualdad.

Ciertamente se ha avanzado mucho en la integración de la mujer en el ámbito social y educativo, pero aún hoy su situación es, y tiene que ser considerada, un problema generado sobre todo porque el ámbito formal, el de la letra y el espíritu de la legislación, se retuerce en la práctica contra aquello mismo que se quiere favorecer: la igualdad de género.

En los últimos tiempos la educación no sólo ha estado aislada del medio social, económico, político y de convivencia, sino que ha dejado de ser su modelo orientador, para plegarse a sus conveniencias. Esto está generando que tanto los planes educativos, como la estructura y funcionamiento de los centros en que se realiza la educación y los organismos ligados a ellos se estructuren intentando producir una relación más activa y fructífera con la sociedad. En esta situación, y aunque la legislación que afecta a las relaciones humanas en la estructura educativo-social establece la igualdad de los géneros, en la práctica los obstáculos que la mujer encuentra para ser aceptada en condiciones de respeto e igualdad con el hombre, la marginación y exclusión que sufre en muchos casos, incluso la búsqueda y producción de situaciones de intolerancia a las que su sensibilidad no puede adaptarse en forma alguna, están conduciendo a generar dos sociedades: una la del hombre, otra la de la mujer.

Como la mujer percibe en el entramado educativo-social de la educación superior que a pesar de todos sus esfuerzos las relaciones humanas no la incluyen, sino que la excluyen, la imposibilidad de asumir esta situación, y la necesidad de buscar soluciones, ha llevado incluso a plantear la conveniencia de volver de nuevo a una educación por géneros. El esfuerzo que la mujer tiene que realizar para conseguir unas

\footnotetext{
${ }^{1}$ En: "España: Resumen informativo de la Plataforma Impacto de Género Ya con relación al sexto informe periódico que presenta España ante el Comité sobre la Eliminación de la Discriminación de la mujer de la ONU". Junio 2009, se señala en su pág. 8: "Valoramos positivamente que en abril de 2008 se creara en España el Ministerio de Igualdad, en Consonancia con la Recomendación CEDAW nº (1998) y la medida 203 de la Plataforma de Acción de Beijing, que incluye las políticas de juventud y de igualdad de género. No obstante, el presupuesto dotado lo consideramos insuficiente (en 2009 ha sido de 80,96 millones de euros que supone tan solo el $0,02 \%$ del gasto no financiero del Estado español).
} 
condiciones que le permitan competir en igualdad, no se corresponde con los resultados que recibe en el ámbito socio-educativo superior. En este ámbito el proceso de igualdad aparece frenado en el acceso a los puestos docentes, investigadores y de organización en función de la idea de que si el esfuerzo femenino no tiene satisfacción se debe a que a la mujer no se le pide realmente ningún esfuerzo, sino que es ella quien se empeña en realizarlo y, por tanto, no tiene por qué tener ningún reconocimiento, pues no se tiene por qué favorecer la realización de una igualdad que no sólo no se pide, sino que incluso resulta molesta e indeseable. Ahora bien, el esfuerzo de la mujer más que entenderse como una lucha con el otro género, tiene que comprenderse como una forma que adquiere la lucha de los seres humanos por alcanzar sus derechos, por ello, no es que la sociedad tenga que pedir un esfuerzo a la mujer, sino que éste le es exigido por la misma necesidad que a todo ser humano le exige realizarse plenamente y como tal.

El segundo género, como lo llamaba Simone de Bouvoir, comenzó a incorporarse al medio socio-educativo-laboral a la sombra y mediante el apoyo del otro. No podía ser de otra forma, ;el mundo era de los hombres! El problema hoy es que se sigue en la misma tesitura, y sólo cuando uno o un grupo de hombres pretenden y buscan la realización de la mujer ésta se produce, pero la mujer o las mujeres, por sí mismas no logran más que la formación de grupos aislados o paralelos sea de investigación o de producción. Sencillamente, la sociedad frena e impide el paso de la mujer a los puestos directivos y ejecutivos, de poder, aunque la unidad y estabilidad social, tanto en sus unidades mínimas (familias), como en la convivencia laboral, resulte perjudicada. Por ello, es necesario fomentar la celebración de reuniones en que se confronten estudios y de las que se origen otros, de manera tenga lugar una atención y seguimiento de la situación que permita abrir vías para avanzar en el logro de la igualdad.

\section{Un análisis cuantitativo de la situación de la mujer en el ámbito socio- educativo superior español}

Una observación de las estadísticas oficialmente publicadas refleja la siguiente situación de la mujer relativamente a la del varón en el ámbito educativo superior.

En las Universidades españolas, tanto públicas como privadas, el porcentaje de matrícula general de la mujer en el curso 2008-2009 es mayor que el del hombre: 
Figura 1 - Matrícula Curso 2008-2009: Porcentaje Relativo de Hombres y Mujeres

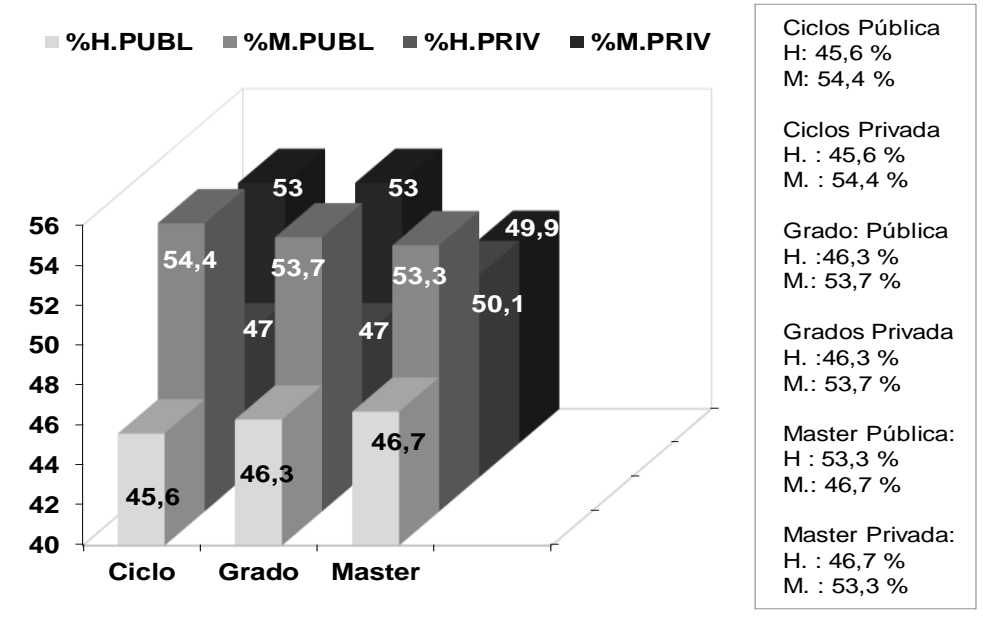

Elaboración propia. Datos I.N.E

Esta misma situación se reitera cuando la estadística refleja el número de Mujeres/Hombres matriculados en $1^{\circ}$ y $2^{\circ}$ Ciclo, Grados y Másteres universitarios, como puede observarse en los siguientes gráficos:

Figura 2 - Relación del Número de Mujeres Matriculadas en $1^{\circ}, 2^{\circ}$ Ciclos de las Universidades, con los Totales (general y parciales): Curso 2008-2009

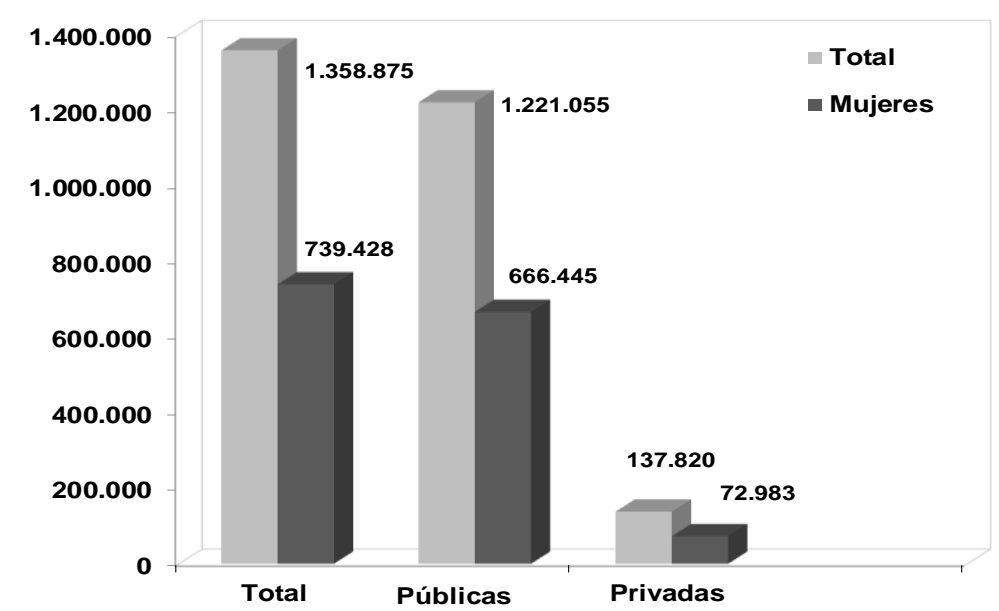

Elaboración propia. Datos Universia 
Figura 3 - Relación del Número de Mujeres Matriculadas en Grados de las Universidades con los Totales (general y parciales): Curso 2008-2009

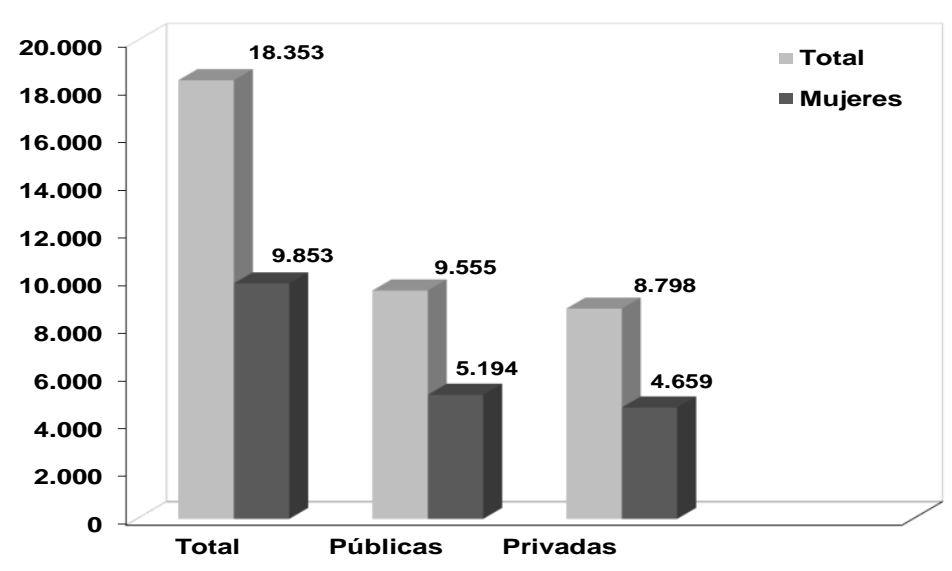

Elaboración propia. Datos Universia

Figura 4 - Relación del Número de Mujeres Matriculadas en Másteres Oficiales con el Número Total (global y parcial) de Alumnos: Curso 2008-2009

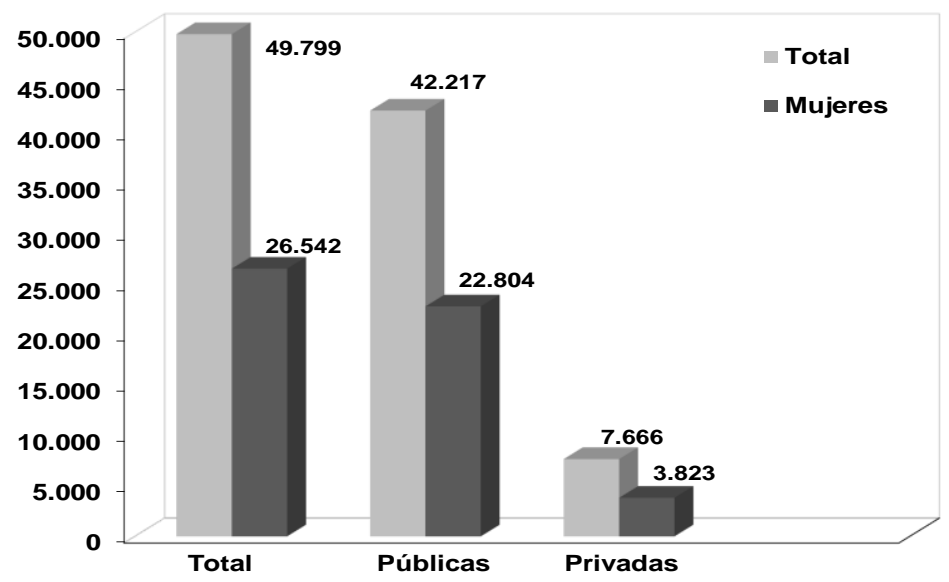

Elaboración propia. Datos Universia

El mayor número de mujeres relativo al de varones se mantiene en la graduación de primero y segundo ciclo de las Universidades y Másteres: 
Figura 5 - Relación del Número de Mujeres Graduadas en $1^{\circ}$ y $2^{\circ}$ Ciclo de las Universidades con el Total (global y parciales) de Graduados: Curso 2008-2009

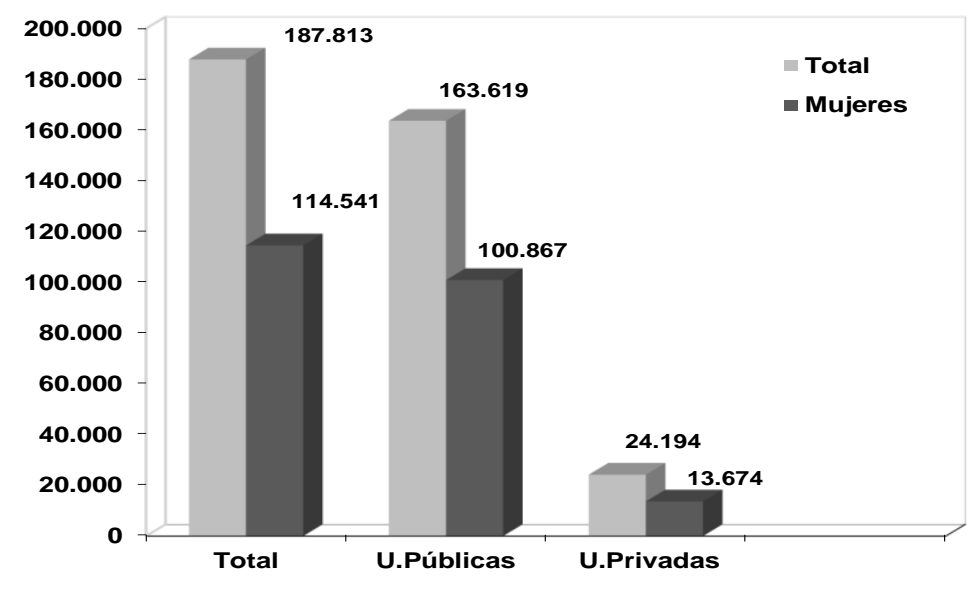

Elaboración propia. Datos Universia

Figura 6 - Relación del Número de Mujeres Graduadas en Másteres Oficiales con el Total de Matriculados (global y parciales): Curso 2008-2009

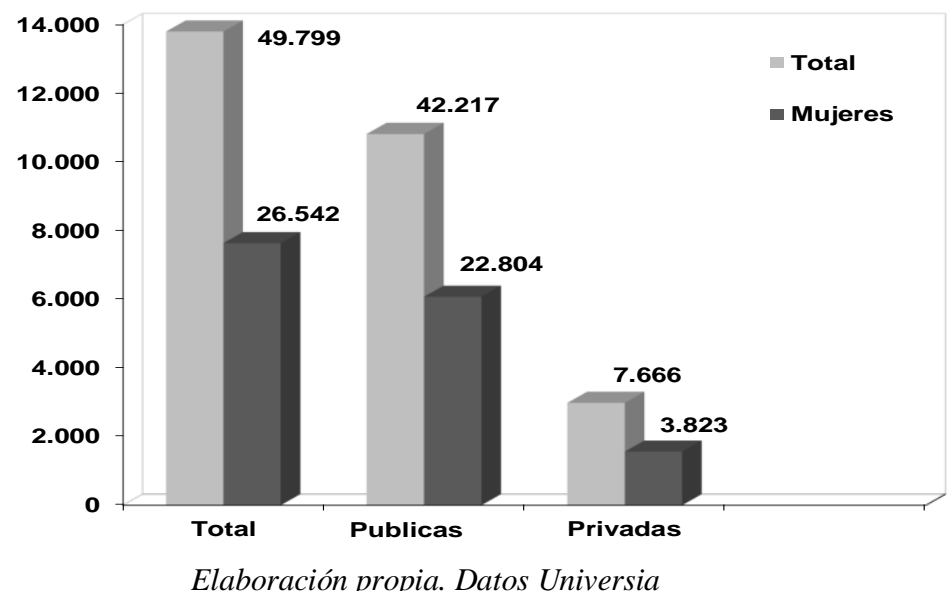

Los porcentajes totales relativos a la Graduación pueden visualizarse en el siguiente gráfico, en el que se muestra también la ausencia actual de titulados en los Grados: 
Figura 7 - Graduación Curso 2008-2009: Porcentaje Relativo de Hombres y Mujeres

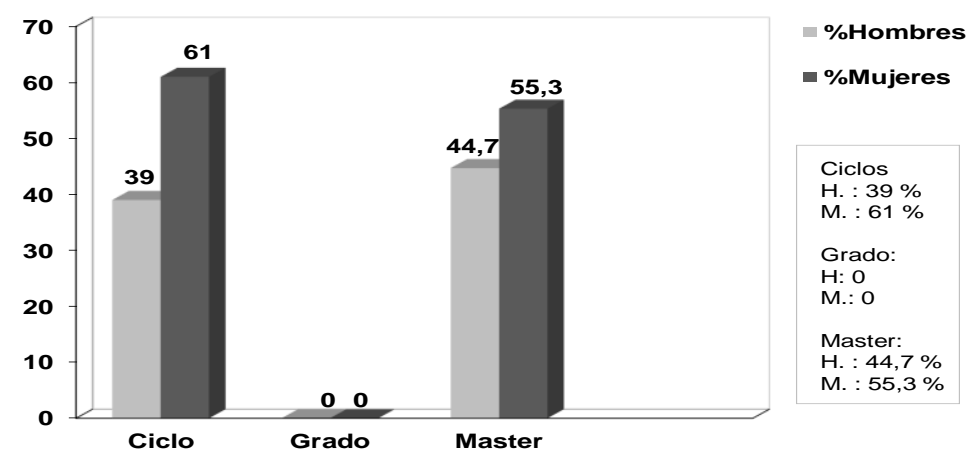

Elaboración propia. Datos I.N.E.

La superioridad numérica de la mujer sigue siendo evidente tanto en la finalización de los estudios, en la matrícula de doctorado, como en la conclusión de las Tesis Doctorales, salvo en la $\mathrm{C}$ y $\mathrm{T}$ en que continúa siendo superior el número de varones:

Figura 8 - Matrícula Doctorado 2008 Porcentajes Hombre/Mujer

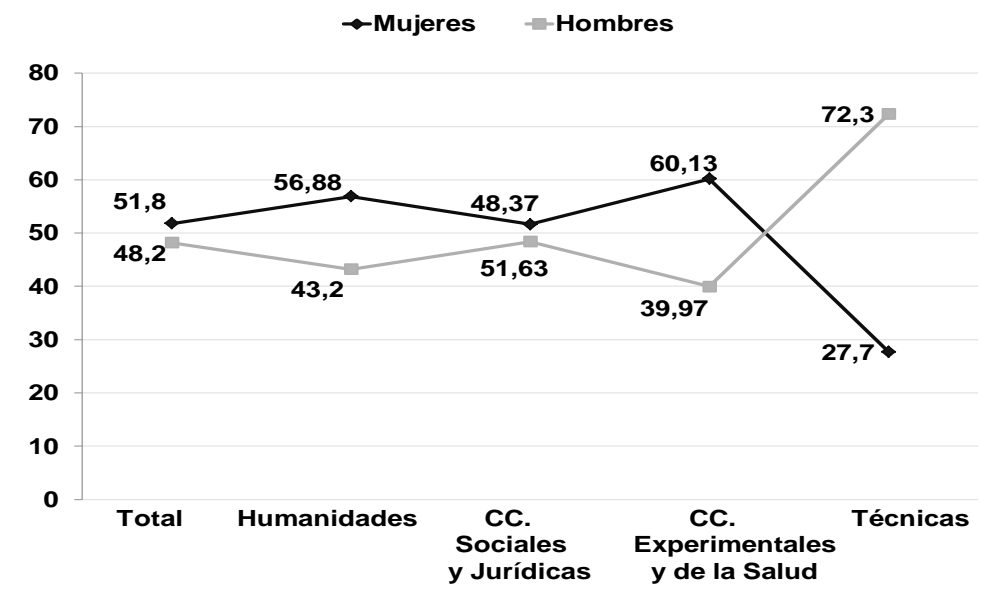

Elaboración propia. Datos I.N.E. 
Figura 9 - Tesis Aprobadas 2008 - Porcentajes Hombre/Mujer

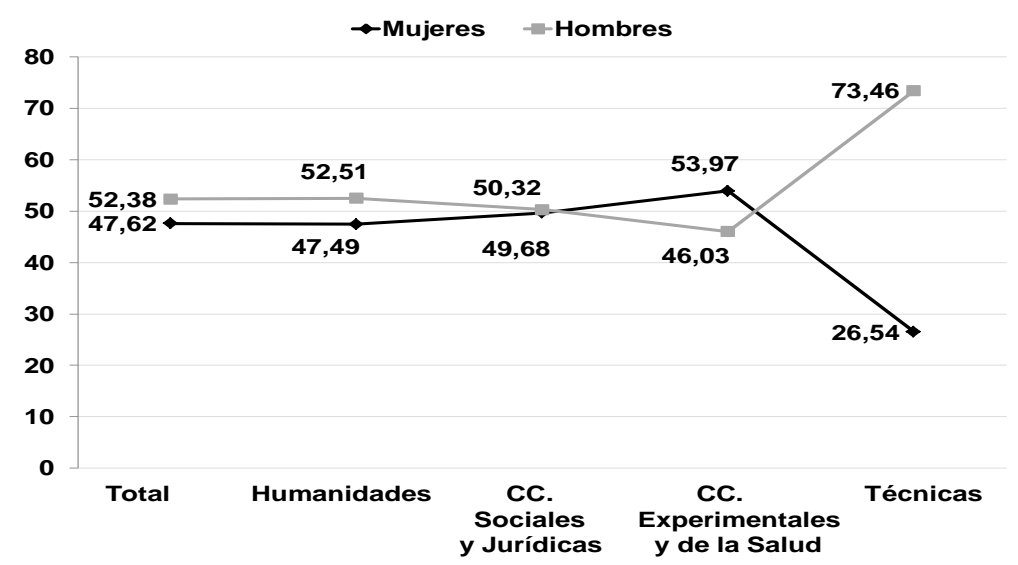

Elaboración propia. Datos I.N.E.

La situación cambia drásticamente en cuanto se consideran las estadísticas relativas al ejercicio de la profesión en la Educación Superior, tanto en lo que afecta a los puestos que permiten la práctica docente como la investigadora. En los cuerpos docentes de funcionarios universitarios, el porcentaje superior es masculino, como se observa en el gráfico siguiente:

Figura 10 - Porcentaje Profesorado Cuerpos Docentes en Universidades Públicas 20072008: Relación Hombre/Mujer

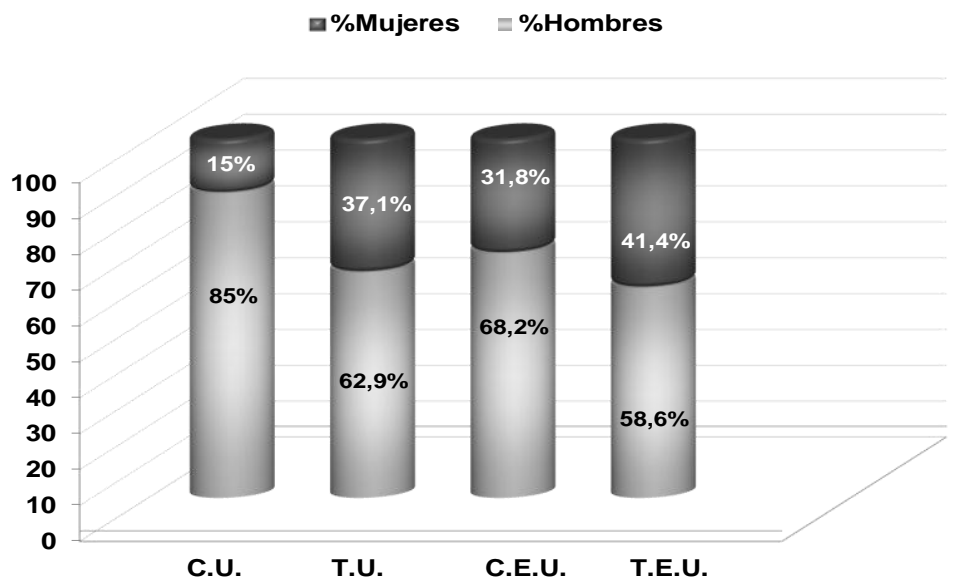

Elaboración propia. Datos I.N.E. 
Lo mismo sucede en los cuerpos docentes de profesorado contratado:

Figura 11 - Cuerpos Docentes y Profesorado Contratado 2007-2008: Hombres/Mujeres

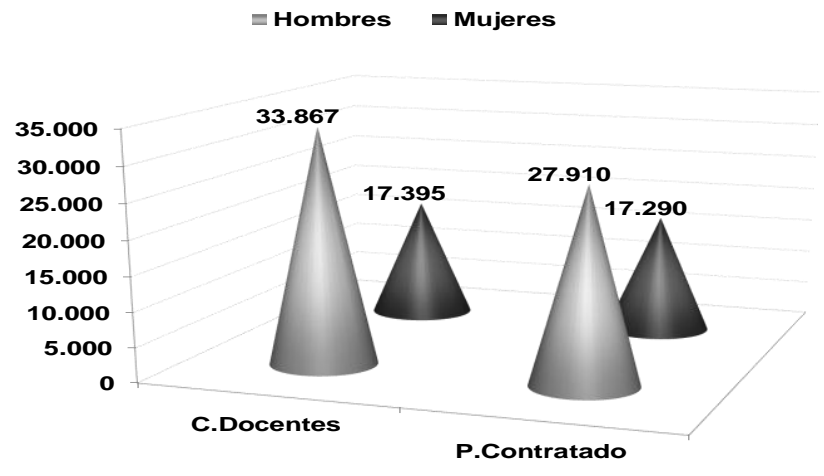

Elaboración propia. Datos I.N.E.

La superioridad numérica del hombre es patente también en los totales de investigadores contratados en el curso 2007-2008:

Figura 12 - Total de Investigadores contratados: 2007-2008

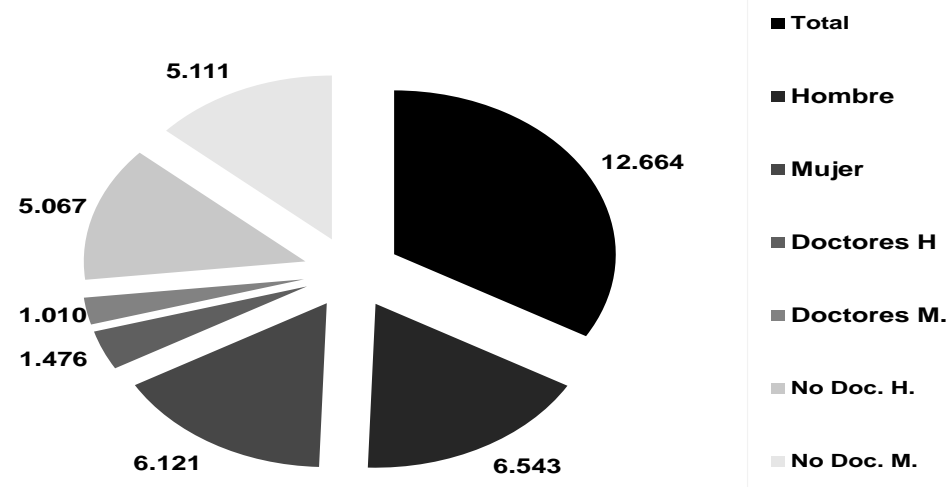

Elaboración propia. Datos I.N.E. 
En el sector de la producción investigadora la superioridad del varón sobre la mujer resulta igualmente evidente, salvo en áreas muy puntuales, como se puede comprobar en el gráfico $\mathrm{n}^{\circ} 13$ :

Figura 13 - Porcentaje de Publicaciones Desagregadas por Género: 2006

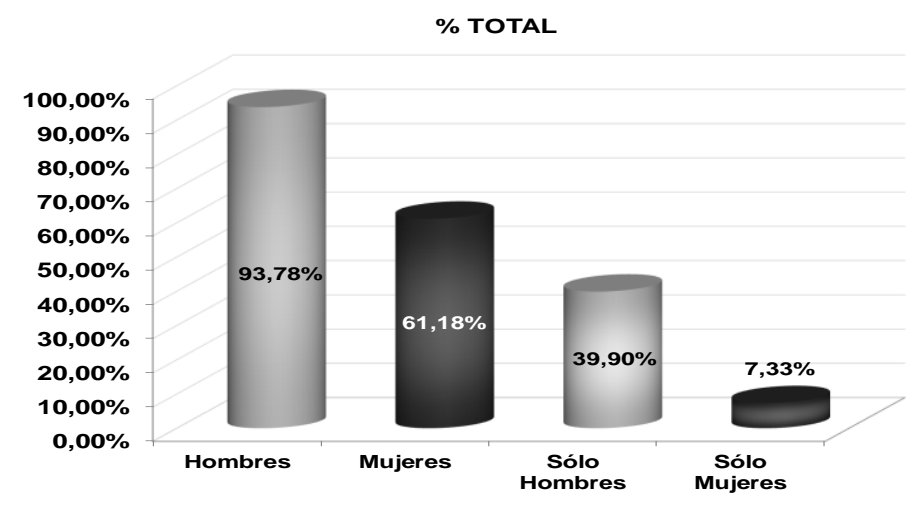

Elaboración propia. Datos F. E. C.Y. T.

En el siguiente gráfico se muestra, en algunas áreas significativas, las curvas relativas de la producción investigadora de ambos géneros. La diferencia, que siempre es favorable al hombre, salvo en Alimentarias, es mucho mayor en el área de las Técnicas:

Figura 14 - Porcentaje de Producción Total según Género: 2006

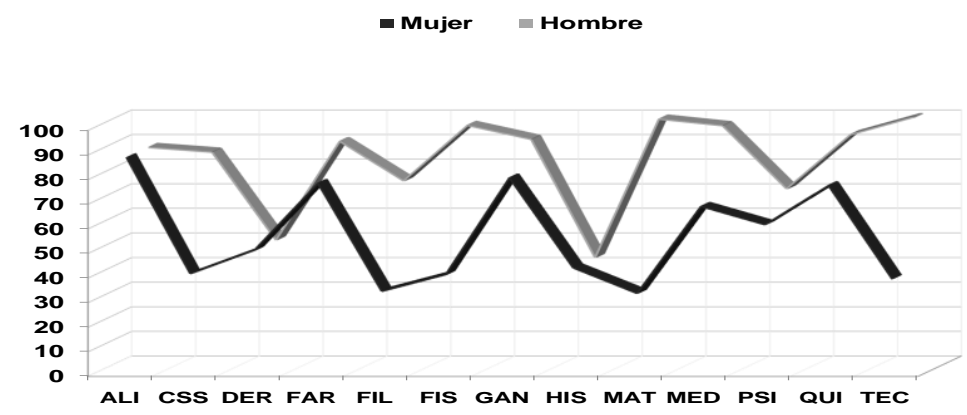

Elaboración propia. Datos F. E. C.Y. T. 
En todo el campo relativo a las publicaciones, las estadísticas son siempre favorables a los hombres. Ahora bien, la mayor diferencia entre hombre/mujer se aprecia en la consideración del potencial investigador de la producción:

Figura 15 - Potencial Investigador de los documentos firmados sólo por hombres y sólo por mujeres: 2006

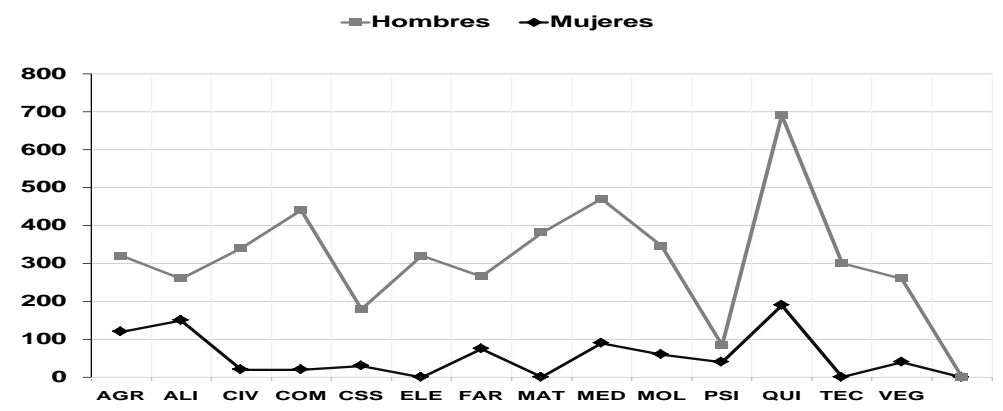

Elaboración propia. Datos F. E. C.Y. T.

En conformidad con los datos que se aprecian en los gráficos anteriores sobre la situación de la mujer en la educación superior española, que no varía sustancialmente por mucho que se profundice, se añadan datos y se pormenoricen los sectores, la conclusión del análisis no puede ser otra que, frente al proceso de aprendizaje, en los sectores relativos al ejercicio profesional en educación superior: docencia e investigación, la superioridad numérica del varón es evidente, y que esta diferencia se transforma en un abismo total abierto en la relación hombre/mujer cuando consideramos los sectores relativos al poder y toma de decisiones relacionados directamente con la educación superior y su productividad. Efectivamente, al considerar los porcentajes relativos a estos contextos encontramos que en el año 2010 en las Universidades españolas hay 10 rectoras (cuatro en las Universidades Públicas y seis en las Universidades Privadas). El número ha aumentado al doble relativamente al año 2006 en que había tan sólo cinco rectoras, pero frente a los sesenta y tres rectores varones la desproporción es evidente: 
Figura 16 - Universidades Españolas 2010: Rector/Rectora

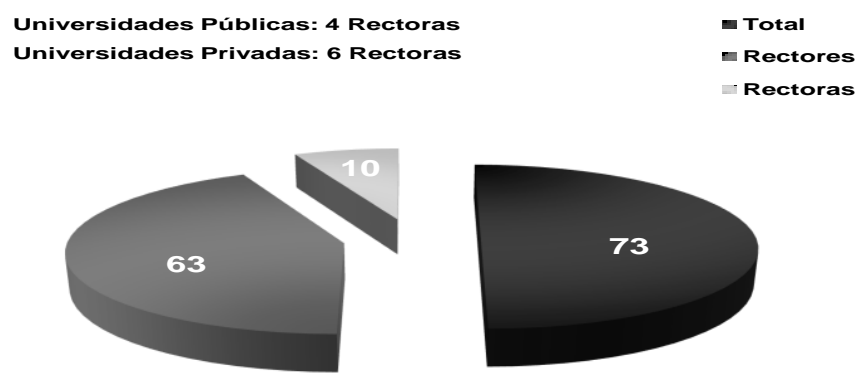

Elaboración propia. Datos I.N.E.

Si se consideran los gráficos en que se representa el alcance de la participación que se concede a la mujer en el contexto social, pero como resultado del desarrollo y culminación de la enseñanza superior, se puede observar que el número de personas premiadas muestra, en todas las áreas, la superioridad del reconocimiento concedido al hombre frente a la mujer:

Figura 17 - Personas Premiadas Según Área y Género: 2009

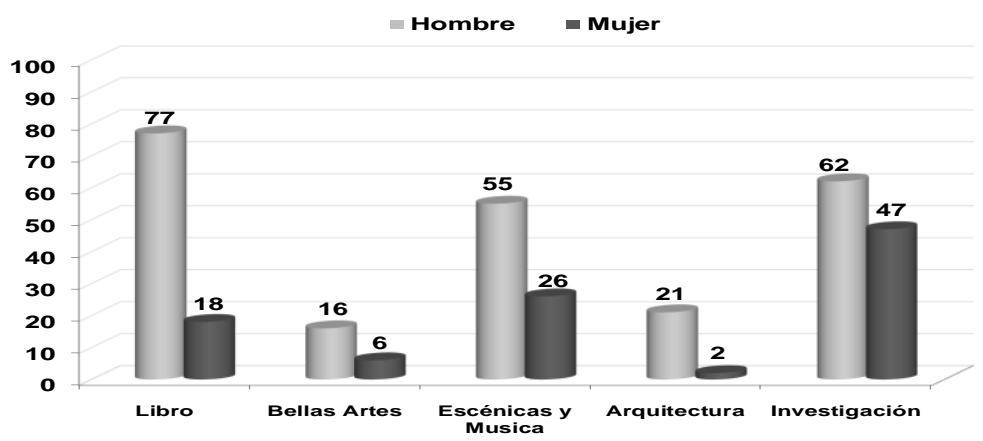

Elaboración propia. Datos F. E. C.Y. T.

De los Doctorados Honoris Causa concedidos en el año 2008 (82 en las Universidades Públicas) las mujeres tan sólo alcanzan el 12,2\%. En las Universidades Privadas se concedieron 15 Doctorados H.C. y, de ellos, ninguno a mujeres: 
Figura 18 - Doctorados Honoris Causa: 2008

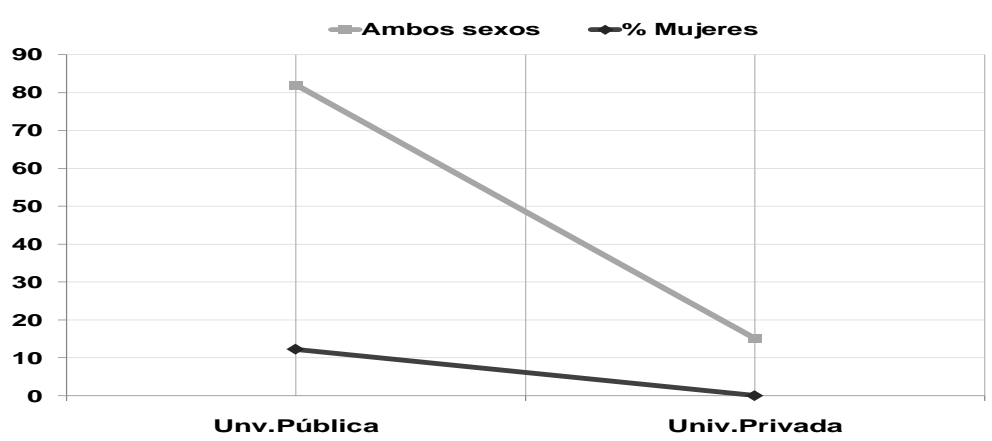

Elaboración propia. Datos I.N.E.2010

El gráfico en que se muestra la correlación del número total de personas y el porcentaje correlativo de mujeres que pertenecen a las Reales Academias en el año 2009, refleja los siguientes datos relativos a la mujer: R.A. de Bellas Artes: 1,7\%; R.A. de Ciencias: 2,3\%; R.A. de CC. Políticas y Morales: 2,6\%; R.A. de Doctores: 12,5\%; R.A. Española: 7,0\%; R.A. de Farmacia: 11,1\%; R.A. de la Historia: 8,6\%; R.A. de Jurisprudencia: 0; R. A. de Medicina: 2,1\%; R.A. de Veterinaria: 10\%:

Figura - Mujeres Académicas Numerarias en las Reales Academias Españolas: 2009

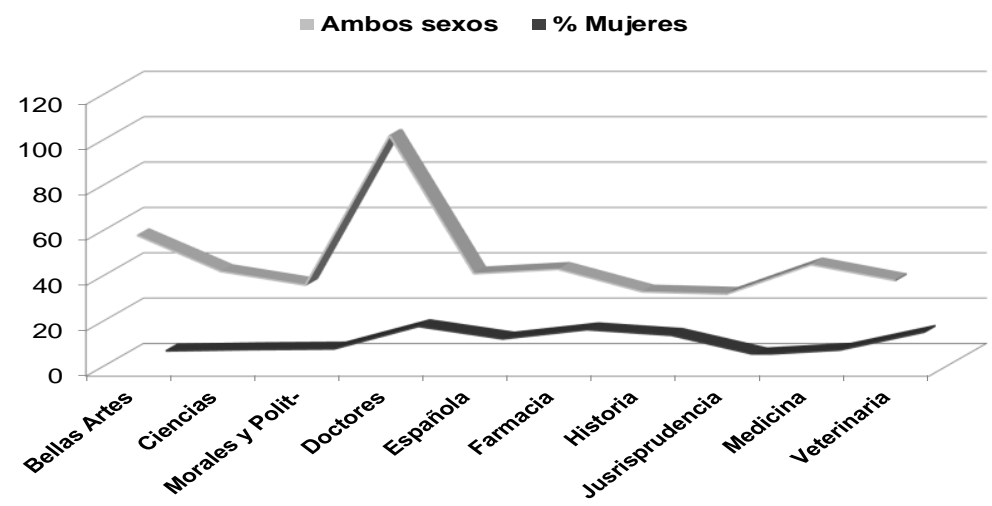

Elaboración propia. Datos I.N.E.2010

En conclusión, en la educación superior la situación de la mujer es buena en el proceso de aprendizaje, pero no en la profesionalización y reconocimientos sociales. Por ello, y en vista del estudio realizado resulta la necesidad de dejar de abordar el estudio 
de la situación de la mujer en la educación superior de forma global, y considerarlo distinguiendo niveles de análisis, entre los que necesariamente hay que atender los siguientes: 1. El acceso a la enseñanza universitaria. 2. El proceso educativo en las universidades. 3. El proceso de formación en investigación. 4. El acceso a los puestos docentes y de investigación. 5. El acceso a los cargos organizativos y ejecutivos. 6. La situación de la mujer en los núcleos sociales de decisión ligados a la educación superior. ${ }^{2}$ Relativamente al estudio de estos niveles es preciso preguntarse y saber de qué depende la situación de la mujer, e intentar vías que la resuelvan de una manera menos forzada que la tan discutida paridad y las cuotas que hasta ahora se promueven.

\section{La lucha de la mujer contra el tiempo es una lucha por los principios}

La situación de la mujer en la educación superior que se visualiza en el análisis cuantitativo anterior muestra una marginación profesional (docente, investigadora y ejecutiva) real y efectiva.

Cuando se atiende en términos generales a la situación de marginación de la mujer se concibe como una lucha contra el tiempo. Pero esta expresión se entiende de una forma abstracta y vacua: hemos empezado tarde..., no hemos tenido tiempo....La pregunta que me formulo, y que trato de responder, es ¿qué operatividad permite esta expresión?

Filosóficamente la noción de contratemporalidad (contretepms) tiene un lugar propio en la filosofía hegeliana, en la que, al hacerse equivalente historicidad y temporalidad, cualquier totalización supone la contracción de la dispersión temporal por una idea (principio). En efecto, contratemporalidad adquiere allí el significado de que la dispersión que proporciona la temporalidad (esto, aquí, ahora), adquiere indefectiblemente una historicidad, es decir, que necesariamente da lugar a una totalización solo posible por la contracción de lo disperso temporal según unos

\footnotetext{
${ }^{2}$ Hoy todos los estudios sobre la enseñanza superior coinciden en señalar la situación positiva que ha alcanzado la mujer en los estudios universitarios y la situación negativa en que se encuentra profesionalmente, pero no se está abordando el análisis distinguiendo niveles y realizando propuestas sobre cada uno de ellos. Todo lo más se señala la situación de inferioridad de la mujer en los cuerpos docentes y contratados. Un estudio reciente es el elaborado en el XI Encuentro Estatal de Defensores Universitarios titulado Igualdad efectiva hombre-mujer en el ámbito universitario - Oviedo, 12-14 de noviembre, 2008.
} 
principios cuya realidad es la de ser contretepms, es decir, la de evitar la dispersión y, por ello, negar el pasado ${ }^{3}$.

Si usamos la noción ya clásica entre génesis y estructura para entender la contratemporalidad, hay que decir que ésta supone la negación de la génesis por la estructura; la negación de lo que ha sido por el lugar que según principios pasan a ocupar en una estructura. De esta forma, si bien es indudable que la génesis proporciona los contenidos que se van a integrar en la totalización estructural, pues los contenidos siempre constituyen elementos temporales, también es indudable que la constitución de la estructura supone regresar sobre esos contenidos en forma contratemporal, es decir, dotándolos de necesidad, eliminando su dispersión ${ }^{4}$.

La contratemporalidad se muestra como Aufhebung, como superación en la que lo hecho, el pasado, es cancelado, asumido como principio e integrado como momento en una totalidad.

El problema de la contratemporalidad que muestra la filosofía hegeliana es que la necesidad de los principios que pueden contraer la dispersión en una totalidad estructurada, no puede considerarse de forma intemporal, pues, la misma superación del pasado al exigir la transformación de la sucesión temporal en secuencia racional, es decir, en secuencia dotada de necesidad, indefectiblemente va a mostrarse también temporal.

Ciertamente, en la superación de lo empírico disperso por constitución de una nueva totalización, los principios pasados no se pierden, no desaparecen, pasan a ocupar otro lugar y a adquirir otro significado: se transforman; pero, entonces, es necesario obtener respuesta a algunas preguntas como las siguientes: ¿de qué forma tiene lugar la necesidad de esta transformación?; más en concreto: ¿qué determina que un principio, y no otro, sea el necesario para dar lugar a la totalización?, preguntas que obligadamente exigen una respuesta dado que en función de esa transformación y del principio que la permite, lo anterior (las situaciones, posiciones y relaciones que favorecían) pasa a ocupar un lugar secundario.

El planteamiento realizado de la contratemporalidad supone que la necesidad de constitución de una estructura que permita una nueva forma de entender y constituir la realidad de la mujer, está dada en el mismo orden empírico, en las acciones mismas. Pero, que ésta tenga lugar de forma efectiva, que se realice, requiere

\footnotetext{
${ }^{3}$ He expuesto esta noción para explicar la reconstitución hegeliana de la Historia de la Filosofía. Véase Lafuente (1986: 31-47).

${ }^{4}$ Escohotado entendió muy bien el problema al considerarlo en el contexto de la Historia de la Filosofía. Ver: Escohotado (1975: 14-15)
} 
poder mostrar y dar cuenta del principio o principios que permiten su racionalización $\mathrm{y}$, al propio tiempo, saber tanto la forma en que los principios dan lugar a la conexión de lo disperso, como saber qué supone su irrupción en el marco social.

Si nos fijamos en la secuencia que la mujer ha desarrollado en su lucha por lograr su libertad y ser reconocida como ser humano, que es el fundamento de lo que se identifica como igualdad, pueden delimitarse tres aspectos básicos: El que afecta a la mujer individual, el que afecta a la mujer como ser social, y el que afecta a la mujer como figura de la Cultura y la Historia. Si nos preguntamos qué principios rigen estos aspectos la respuesta no es otra que la siguiente: El primer aspecto está regido por principios naturales, pues supone la lucha de la mujer como ser natural; el segundo por principios sociales, pues afecta a la lucha de la mujer como ser social, y el tercero por principios de universalización que permitan su caracterización como ser cultural e histórico.

Los principios rectores de la producción de estructuras determinantes de las diversas formas de orden (natural, social, histórico-cultural), han llevado a que la situación de la mujer tenga que ser la de un ser en lucha por sus derechos. Su exigencia de igualdad es la reclamación de unos principios que hagan realidad su derecho a ser reconocida como ser humano.

Como ser natural la lucha de la mujer es una lucha contra los principios estructuradores vigentes todavía hoy, pues, en conformidad con ellos, por ser sus condiciones físicas diferentes de las del otro sexo, ha sido considerada como inferior, por ejemplo, porque no tiene igual fuerza física, porque su organismo tiene la capacidad de concebir otro ser humano, etc. Como ser social, porque la impiden lograr superar el principio de minoría de edad, parejo a la carencia derechos, bajo el que se la enjuicia psicológica y moralmente y lograr que los derechos humanos sean realmente iguales en educación, en trabajo, remuneración, y en el acceso a responsabilidades sociales y jurídicas, etc. Como ser cultural e histórico, la lucha de la mujer se desarrolla en el sentido de conseguir ser considerada en igualdad con relación a los principios de autoridad y poder.

Ahora bien, tenemos que considerar que estas tres determinantes: física, social, e histórico-cultural, sólo constituyen el esquema básico para entender la situación de la mujer, pues es preciso poner de relieve el hecho que si bien por sí mismos constituyen esferas conceptualmente diferenciables de forma absoluta, en lo que afecta a trabajar con la realidad de la mujer y a lograr una evaluación y valoración sólida, hay que tener en cuenta que históricamente la consolidación de un orden 
estructural depende de cómo se entiendan las relaciones entre los principios rectores de estas tres esferas, y a cuál se otorgue el primado.

En lo que afecta a la situación educativo-social de la mujer considerar que un orden (contratemporalidad) depende de qué principio se consolide como unificador de la multiplicidad, requiere alcanzar a saber qué principio es capaz de ordenar la acción temporalmente dispersa de la mujer de forma que la oriente con el objetivo de lograr superar la marginación profesional efectiva en la que éste género se encuentra actualmente.

Este planteamiento conduce a considerar que hoy ya no son suficientes unos principios que proclamen una igualdad meramente formal, sino que es necesario encontrar aquellos que muestren su valor y eficacia en el ámbito socio-educativo y permitan la universalización de la realidad que corresponde a la mujer. En lograr el reconocimiento y aceptación de la universalización de su acción reside el gran problema de la igualad que la mujer reclama.

\section{Alcance del principio ilustrada de educación igual y generalizada}

Como en los principios existe una jerarquía, se buscan los principios generales para apoyar los particulares, pero esta extensión de los principios es la que permite valorarlos a ellos mismos, mostrar su alcance.

Cuando se invoca el principio ilustrado de generalización e igualdad educativa como garante de igualdad de oportunidades, de situaciones y relaciones sociales, parece que los cielos se abren con la promesa de una nueva aurora. Esta esperanza nace porque se considera que toda condición beneficiosa humana y socialmente tiene su principio rector en la educación. Entonces se invocan lemas históricos como el baconiano saber es poder, pero, sobre todo se vuelve la mirada al modelo gestado por la Ilustración, cuyo fundamento se halla precisamente en la necesidad de superar la minoría de edad, pues la educación puede conseguir hacer realidad la unión saber-pensar-poder-decidir por-sí- mismo (autonomía) y no por principios ajenos (sapere audere).

Esta idea, se constituyó en principio y meta de la educación, pues se consideró que la importancia de la educación no sólo residía en refinar al hombre hasta llevarle a la contemplación de la verdad, como ya había planteado el mundo griego, sino sobre todo en permitir elevar el nivel en que se desarrolla la vida de la 
generalidad de los hombres. Por ello, en este principio se apoyaron los ilustrados para pedir una educación pública: igual y general.

Lo que hace especialmente atractivo este principio kantiano, que ha pasado a la historia como el lema ilustrado por excelencia, es precisamente su condición de universalidad fundada en su capacidad para establecer la conexión de las tres determinantes humanas: física-socio-cultural-histórica. Por ello, cuando se pone de relieve este principio parece que también en lo que afecta a la mujer se están reclamando medidas efectivas que permitan la superación de la situación de negrura y marginación que el proyecto de educación roussoniana consideraba apropiada para ella, la compañera ideal (Sofía, la futura compañera de Emilio).

Hombre y mujer tienen características comunes como especie, según Rousseau, pero como en su forma de entenderlas resultan meramente formales, aquella noción queda sin consistencia: es una abstracción. La realidad de la mujer no puede considerarse sólo como una diferencia que se resuelve en una apariencia de más o menos. ${ }^{5}$ La mujer es un sexo diferente al hombre, y toda diferencia determinable se debe al sexo, contrariamente a lo que sucede con las semejanzas y diferencias generales cuyas consecuencias no pueden conocerse. Pero, como estas diferencias unidas a la diferencia de sexo se concretan en características morales distintas a las del varón, la naturaleza de la mujer se muestra evidentemente diferente y, aún más, inferior a la del hombre. ${ }^{6}$

Según Rousseau, la naturaleza psíquica de la mujer no llega a ser espiritual, no posee, ni puede tener, un alma (razón) individual, sólo tiene un alma común. En su naturaleza no se halla inscrita la ley moral, y lo más que se puede lograr mediante la educación es que su moralidad pase a alcanzar la racionalidad de su marido. Por ello, Rousseau, al establecer la educación como conexión que puede variar las carencias de que adolece física y psíquico-espiritualmente la mujer, entiende que lo único que se

\footnotetext{
${ }^{5}$ La constitución de la mujer, su figura y necesidades solo difieren, según Rousseau, en más o menos respecto del hombre: "En tout ce qui ne tient pas au sexe, la femme est homme...ils ne diffèrent entre ceux que du plus au moins" (Rousseau, 1964: 445).

6 "Sophie doit être femme comme Émile est homme, c'est-à-dire avoir tout ce qui convient à la constitution de son espèce et de son sexe pour remplir sa place dans l'ordre physique et moral...En tout ce qui tient au sexe, la femme et l'homme ont partout des rapports et partout des différences [...] Par l'anatomie comparée, et méme à la seule inspection, l'ont trouve entre eux des différences générales qui paraissent ne point tenir au sexe; elles y tiennent pourtant, mais pour les liaisons que nous sommes hors d'état d'apercevoir: nous ne savons jusqu'où ces liaisons peuvent s'étendre; la seule chose que nous savons avec certitude est que tout ce qu'ils ont de commun est de l'espece, et que tout ce qu'ills ont de différent est du sexe...Ces rapports et ces différences doivent influer sur le moral [...]"'(Rousseau, 1964: 445-446).
} 
puede conseguir es que aprenda a seguir el principio moral que por naturaleza (físicoespiritualmente) tiene inscrito el hombre.

En el proyecto educativo-ilustrado que Kant (1983) expone en su Pedagogía, mantiene que sólo la educación puede promover la igualdad real entre los hombres, y que ella es la base para que los individuos y los Estados puedan realizarla. También sostiene que conseguir la igualdad de los hombres tiene que alcanzar a su forma de vida, lo que sólo puede lograrse si se desarrolla de forma cosmopolita, y no limitada a una sociedad o a un Estado, por lo cual la educación tiene que extenderse a toda la humanidad, pues su preocupación tiene que ser el bien universal, condición que considera necesaria para que haya un progreso real (Kant, 1983: 36).

$\mathrm{Si}$ cuando se atiende al proyecto roussoniano resulta evidente que el alcance de sus principios (su límite) se encuentra en proclamar la necesidad de la educación para el varón como forma de lograr su excelencia, y en intentar mejorar la de la mujer tan sólo en tanto puede contribuir a resaltarla, parece esperarse del proyecto educativo kantiano algo más favorable a la condición humana de la mujer, a la equiparación social de ambos sexos. Pero, como Kant al considerar la educación presenta sus principios filosóficos aplicados al modelo gestado en Europa, entendiendo que es el único fructífero pues todos los otros pueblos son salvajes, sus tesis educativas limitan el alcance de aquellos constriñéndolos a la existencia de un modelo educativo, el europeo, que considera el único que puede cumplir con los objetivos educativos de mejorar la vida, las relaciones humanas, la sociedad y los Estados. Los hombres no europeos tendrán que adecuarse a este modelo, el único que promoverá realmente el progreso $^{7}$.

En este contexto, cuando Kant considera el problema sostiene que la naturaleza femenina no puede adecuarse al modelo europeo de educación, pues si su carácter no puede alcanzar autonomía moral, sino todo lo más formación en el arte de agradar, su pensamiento sólo puede servir para conducir de forma razonada, pero delicada, a los hombres. Como para Rousseau, para Kant la unidad y armonía social será posible si la educación de la mujer se realiza en función de lograr que la excelencia del varón resulte más patente y sea mejor valorada, es decir, si la mujer se

\footnotetext{
7 "Se ve también entre los salvajes, que aunque presten servicio durante mucho tiempo a los europeos, nunca se acostumbran a su modo de vivir; lo que no significa en ellos una noble inclinación a la libertad, como creen Rousseau y otros muchos, sino una cierta barbarie; es que el animal aún no ha desenvuelto en sí la humanidad" (Kant, 1983: 31).
} 
limita a cumplir con la función de agradar y servir para resaltar la perfección del varón ${ }^{8}$.

Si la consideración ilustrada no acoge en su proyecto un desarrollo educativo que favorezca la posición social de la mujer, menos resulta éste de la concepción freudiana, que es la que sirve de apoyo a la forma de entender, constituir y justificar el orden, y la forma de las relaciones sociales en gran parte de la Edad Contemporánea. En la forma en que se ha desarrollado, esta concepción condena a la mujer a ser un ser inferior al hombre al establecer la condición sexual como base de interpretación de todo lo que afecta a lo social, y sostener de forma abierta que es la condición física de la mujer, entre otras razones porque sus órganos sexuales no llegan ni pueden llegar (a diferencia de los niños varones) al desarrollo que alcanzan los de los hombres, la que determina su imposibilidad de superar la condición de minoría de edad. La mujer no alcanza el desarrollo genital del varón, y esta diferencia, que se interpreta como falta de desarrollo, lleva a considerar a la mujer como un ser débil, supeditado, sometido, y dependiente de otro. No puede lograr acciones encaminadas a establecer una sociedad libre, lo mismo que no puede constituir un equilibrio entre el yo y el otro.

En conclusión los ilustrados, excluyen de su proyecto educativo a la mujer como ser con valor universal, por tanto, como ser humano con pleno derecho al considerar que es incapaz de constituir principios morales propios, con autonomía y, con ello, limitan el alcance universal de sus principios filosóficos. ${ }^{9}$ Para el pensamiento ilustrado la mujer no es, ni puede ser, un ser libre, pues no puede establecer principios universales. Esta tesis alcanza su cumbre en la obra freudiana en la que ya no se atiende sólo a la constitución de principios, aunque estos sean ligados a la acción, sino a los resultados de la acción: la acción de la mujer no puede ser el fundamento de una sociedad libre.

\footnotetext{
${ }^{8}$ En general, todo este tema que afecta a las exclusiones que tienen lugar en la aplicación de unos principios filosóficos puede considerarse con relación al desarrollo de la filosofía de la Otroriedad cuya tesis básica puede resumirse en sostener la inexistencia de universalidad filosófica, y considerar que esta idea es un simple reclamo que oculta y encubre siempre un geocentrismo de base. Sobre este tema remito a la obra de Enrique Dussel.

${ }^{9}$ Considero que los principios filosóficos ilustrados kantianos siguen siendo totalmente válidos, pues la limitación de su universalidad no procede de ellos mismos, sino de que su aplicación pedagógica se basa en estudios biológicos y médicos insuficientes. En este sentido, hay que sostener que hoy sólo es aceptable un uso universal de estos principios. Con estas afirmaciones no pretendo justificar a los ilustrados, ni salvar sus insuficiencias, sino validar el uso universal que actualmente puede y es necesario hacer de principios como los de Pestalozzi y Kant.
} 


\section{Género y necesidad de un nuevo principio estructurador de la acción socio- educativa-superior}

Nadie niega hoy el valor y la necesidad de una educación igual y generalizada, pero ya es preciso considerar este imperativo como una idea que, en sus realizaciones prácticas, reclama una reflexión que busque los principios capaces de estructurar las acciones dispersas y producir unos resultados satisfactorios. ${ }^{10}$ En la actualidad, el principio que se considera con capacidad para aglutinar las diversas perspectivas y condiciones exigidas en educación es la calidad.

Los diversos organismos rectores de la educación, y de forma muy similar en los países que han alcanzado un desarrollo medio y alto, han sentado los principios, y medios para entender qué puede ser objeto de cualificación y de qué forma. En la búsqueda de los medios que permiten lograr y evaluar la calidad confluyen las agencias evaluadoras de la calidad, y las entidades educativas superiores (Universidades, Centros de Investigación, Centros de Formación) que tienen que manifestar en su presentación ideas, intenciones y objetivos que las signifiquen de forma especial, pero con los que tienen que cumplir. Con ello quiero significar que hoy todo el proceso en educación, desde la matricula, pasando por el acceso a los puestos de trabajo, hasta la determinación de los cargos ejecutivos, está presidido por el principio de calidad.

Ambos sexos inician y terminan sus estudios regulados por este principio y les son aplicados los mecanismos evaluadores en el período de formación en igual forma. La sociedad favorece esta formación e incluso la exige. ¿Por qué entonces se produce un cambio significativo en el acceso a las profesiones?; ¿por qué la variación sustantiva se centra en la menor ocupación de puestos de trabajo y de responsabilidad por la mujer?

Puede afirmarse con seguridad que la razón de que los puestos de trabajo y de responsabilidad sean ocupados mayoritariamente por hombres depende de una variación

\footnotetext{
${ }^{10}$ Hay que tener en cuenta que la acción de la mujer tiene que considerarse doblemente dispersa, pues frente a la de otros seres humanos marcados por la clase social o el grupo racial (negros, indios, etc.), cuyas acciones son dispersas pero con peso social específico como grupo, la acción de la mujer nunca fue considerada históricamente como acción de un colectivo, sino que siempre se la excluyó del ámbito público y se la relegó a ser un átomo social al servicio exclusivo del cuidado de otros seres humanos (padres, hijos, marido, etc.). Un dato en este sentido es que Marx ni siquiera considera el caso de la mujer al plantearse las clases sociales en el Dieciocho Brumario de Luis Bonaparte.
} 
muy importante en la forma de entender y aplicar el principio que sustenta la calidad: la competitividad.

Ciertamente estamos en una sociedad altamente competitiva, en la que desde que nacemos estamos insertos en competiciones que van desde la casa, los zapatos, etc., que podemos adquirir, y permiten nuestra apariencia, hasta los puestos de trabajo que podemos ocupar, el dinero del que podemos disponer, etc.

En el proceso de aprendizaje la violencia y agresividad que supone la competitividad está paliada, dado que las evaluaciones son resultado de unos procedimientos que se centran en el rendimiento individual del alumno/a, y éste en el cumplimiento satisfactorio de los objetivos que le son fijados públicamente. Por el contrario, en el campo profesional la competitividad no se centra sólo en el acoplamiento de las aportaciones de la persona a los requisitos establecidos (que siempre se exige), sino sobre todo en que los evaluadores (persona o personas que han de dirimir el caso) decidan que las condiciones del aspirante son acogidas por los principios de evaluación como debe ser.

La introducción de este principio que orienta la interpretación de la calidad abre la profesionalización al orden práctico-moral en el que la forma de dirimir la diversidad de factores que confluyen en la decisión se interpreta y hace equivalente a otro principio: la fuerza mayor. ${ }^{11}$ Pero, que en última instancia dependa de éste principio "tener acceso a...", la resolución relativa a un puesto de trabajo, está condicionando muy negativamente la situación profesional de la mujer, pues éste principio no es inocente, no depende de condiciones abstractas existentes en un lugar de nadie y de todos, pues lo que debe ser como fuerza mayor siempre está circunscrito por determinantes tradicionales y sociales. Y, tradicional y socialmente siempre se ha

${ }^{11}$ Cómo entender la fuerza mayor es un tema considerado por Aristóteles. En la Gran Ética presenta la fuerza mayor como necesidad extrínseca y coactiva que puede afectar a las acciones de todos los seres, tanto animados como inanimados. La fuerza mayor obra como causa extrínseca coactiva que obliga a actuar a los seres incluso contra su naturaleza y voluntad. Cuando, como se ha venido haciendo, se entiende que sólo la unificación físico-moral permite hablar de ser humano (anthropos), y se sostiene que esta unidad sólo se puede adscribir positivamente al género masculino, esta atribución obra como causa extrínseca coactiva sobre toda valoración, es decir, como fuerza mayor. Esta atribución, que es la que lleva a mantener que a la mujer no se la puede considerar igual que al hombre profesionalmente, sólo depende de condiciones establecidas por usos y costumbres tradicionales, pero que obran como si fueran causas universales, cuando más bien lo que impiden es la libertad de acción que le corresponde como ser humano, como ser racional (inteligente), pues, hay que tener en cuenta que como señala Aristóteles: "[...] no hay fuerza mayor en aquellos seres cuya causa activa es intrínseca a ellos mismos", Aristóteles: Gran Ética, Libro I, Cap. 14, 1188a/1188b, en: Aristóteles (1973: 13251326). No existe razón alguna, salvo las que dependen de las apreciaciones educativas tradicionales, para considerar a la mujer excluida de poseer causa activa propia. 
considerado al hombre como más eficaz y eficiente, más capaz, más seguro, más incisivo, más penetrante, más resolutivo, más aglutinador de opiniones, más capacitado para formar y organizar equipos, etc. El más tampoco es inocente, sino una espada de Damocles que pesa sobre la mujer, pues supone una calificación radicalmente contraria a los valores que social y tradicionalmente se le adscriben.

Es preciso explicar algo más la exposición anterior. El principio prácticomoral no es un principio social, sino el principio que constituye esencialmente los valores. De él depende la fijación y adscripción de valores. No es un principio abstracto, un principio general, sino que nace de buscar, entre la diversidad de principios, la razón que permite entender la realización de la acción de la mejor forma. Por ello, el principio práctico-moral delimita absolutamente lo que ha de considerarse ineludiblemente como valor y lo que no tiene porqué ser entendido así. ${ }^{12}$.

No es el principio tal cual: el deber ser como razón que dirime la calidad de las cosas y acciones, sino el uso que tradicional y socialmente se hace de este principio (no la condición esencial del principio) al entenderse como fuerza mayor, el que divide los valores en dos campos y adscribe todos los considerados positivos profesionalmente al hombre y no a la mujer. En esta dicotomía los valores femeninos se consideran no-valores (valores negativos) en el ámbito de lo público, y valores (positivos) en el ámbito privado.

Ahora bien, ¿por qué profesionalmente se valora más (por ejemplo) la penetración que la precisión?, ¿por qué se considera que es mejor ser más capaz de que ser más cuidadoso con?, ¿por qué se adscriben los primeros al hombre y los segundos a la mujer?, ¿por qué se dividen las profesiones en femeninas y masculinas siguiendo estas dicotomías? Que profesionalmente se prefieran los primeros a los segundos se sigue de que laboralmente sólo se aprecian los resultados inmediatos que se juzgan como los más beneficiosos posibles, y se supone que lograrlos exige un control del proceso laboral de arriba abajo, en forma piramidal, y que en la cúspide de la pirámide los valores adscritos a las mujeres desaparecen, y no es que sean invisibles, es son indeseables.

Por esto, la igualdad educativa no puede plantearse hoy simplemente como la necesidad de que la educación sea generalizada, hay que partir de que ésta exigencia es

\footnotetext{
${ }^{12}$ Un pasaje significativo para el tema es aquél en que Aristóteles se pregunta de qué depende que se considere la fortaleza (el valor) como un bien. Clasifica y examina las diversas formas de entender el valor, y llega a la conclusión de que es considerado como un bien, no cuando por experiencia se sabe cuándo y como ser valiente (lo que es propio del valor que corresponde y se adscribe al soldado), sino cuando se piensa que se tiene que ser así: "Podemos definirlo simplemente diciendo que es fuerte cualquiera que no liga su fortaleza a ninguno de estos incentivos, sino que es valiente o fuerte porque piensa que es noble y digno ser así, y es así tanto si está solo como si está acompañado", Aristóteles: Gran Ética, Libro I, Cap. 20, 1190a/1190b, en: Aristóteles (1973: 1330).
} 
ya indiscutible y plantearse cuál es su mejor realización. Cumplir con este supuesto, requiere e implica saber cómo realizarla (cuestión de método); en este sentido hay que partir de que la igualdad educativa tiene que basarse en la aceptación de una pluralidad de valores $\mathrm{y}$, en lo que afecta al género, en la necesidad de lograr la igualdad de los valores que la sociedad entiende como propios de ambos géneros.

Pueden aportarse numerosos ejemplos: mientras en el contexto actual de valores se entiende que formar un equipo equivale a controlar a cada uno de sus miembros, en el contexto de los valores alternativos en que el cuidado pasa a primer plano, formar un equipo será equivalente a establecer un diálogo entre sus miembros, a escucharse unos a otros.

La acción a tomar y los resultados a obtener, no tienen por qué depender de una imposición en la que nadie se reconoce, y que nadie quiere asumir responsablemente, sino ser resultado de un diálogo en el que se sustituya la fuerza mayor por la aceptación de la mejor propuesta. Sustituir el principio de la fuerza mayor por el principio del diálogo, puede permitir romper con la estructura monolítica de los valores que depende de la relación deber-ser-tradición-fuerza-mayor (= mujer inferior), y lograr la aceptación de los valores del otro.

La mujer no puede renunciar a su condición; su exigencia es que ésta: física, social, y culturalmente, sea considerada como un valor positivo igual que lo es la condición masculina en estos ámbitos. La lucha de la mujer en el campo profesional de la educación superior tiene que tener como primer objetivo, que el principio práctico (como debe ser) encuentre su realización más apropiada en los valores que le son propios. Lograrlo no es una tarea individual, ni grupal, sino de toda la sociedad, y en concreto de las instituciones docentes y de todos los docentes, pues exige que a la mujer se la prepare (motive e incentive) desde el comienzo de su aprendizaje para ocupar puestos de poder (organización, dirección, etc.). Pero, es en la acción de cada individuo en la que cobra realidad esta lucha.

Lograr igualdad en el campo profesional de la educación superior supone que la atención educativa que se preste a la mujer y que oriente el proceso educativo sea, desde su inicio, la que corresponde a un futuro líder, o de forma más expresa, no la que considera su educación como un medio para dar lustre y esplendor a una unidad social (matrimonio, parejas de hecho, reuniones sociales en general), ni tan sólo como plataforma para lograr medios de subsistencia, sino como la preparación de un sujeto para hacer que sus valores adquieran un valor indiscutible en los puestos profesionales: docentes, organizativos y ejecutivos. 
Si la educación puede conducir a que los valores del otro sean aceptados, será porque se logre que la mujer se equipare cultural e históricamente al hombre, es decir, porque se la considere un ser cuyos principios son tan universalizables como los de los hombres, y sus acciones tan encaminadas a establecer y mantener la libertad como las de los hombres, es decir, porque la mujer logre ser considerada en igualdad con el otro sexo.

Considero que en lograr que se abra este camino, que puede permitir encaminar la educación a la realización de un orden social más equitativo, dotado de mayor objetividad en la selección y valoración de sus profesionales, y a que se progrese en su mejor desarrollo, es en lo que tiene que consistir la feminización de la universidad, que ahora tan sólo está lograda en lo que respecta al proceso de aprendizaje.

\section{Conclusión}

En muy poco tiempo se ha hecho mucho por lograr la igualdad de la mujer, hasta el punto que su acción se ha llamado la revolución silenciosa. Pero, como se pone de relieve en los análisis realizados, queda mucho por hacer, y casi todo en el campo en de lo educativo-social (puestos docentes, de organización y de decisión), en el que la mujer vuelve a encontrarse con la oposición de los principios que permiten el acceso y rigen la selección de quienes los ocupan.

Evidentemente, no sólo es una cuestión de principios, también es necesario que se produzcan acciones que den lugar a variaciones sustantivas en todos los niveles sociales. Se dice que es la mujer quien tiene que cambiar sus hábitos, forma de organización, etc., realmente lo que la mujer tiene que conseguir es que varíen las condiciones con que la sociedad la marca continua y sistemáticamente: porque quiere trabajar, porque quiere tener hijos, porque quiere tener una casa, porque...., toda la responsabilidad social y moral es de la mujer porque quiere.

La sociedad tiene comenzar a estructurarse pensando que es el lugar de convivencia pública de ambos sexos, y que en una sociedad democrática ambos tienen que tener igualdad de condiciones para acceder a los puestos de trabajo, organizativos y ejecutivos. Tomar conciencia de estos problemas que constituyen el gran obstáculo para evitar la exclusión permanente de la mujer, saber en qué residen, sí es una cuestión de principios, y lograr su solución es una lucha por los principios, pues sólo mediante ellos puede adquirirse la conciencia sin la que no podrán proponerse ni desarrollarse acciones que favorezcan la igualdad real. 


\section{Bibliografía}

- Aristóteles (1973): Obras. Madrid: Aguilar.

- Escohotado, A. (1975): De pysis a polis. La evolución del pensamiento filosófico griego desde Tales a Sócrates. Barcelona: Anagrama.

- Kant, I. (1983) Pedagogía. Madrid: Akal

- Lafuente, Ma I. (1986): Teoría y metodología de la Historia de la Filosofía. León: Universidad de León. Secretariado de Publicaciones.

- Rousseau, J. J. (1964): Émile ou de l'éducation.París: Garnier. 